\title{
An Experimental Downlink Multiuser MIMO System with Distributed and Coherently-Coordinated Transmit Antennas
}

\author{
Dragan Samardzija, Howard Huang, Reinaldo Valenzuela and Theodore Sizer \\ Bell Laboratories, Alcatel-Lucent, \\ 791 Holmdel-Keyport Road, \\ New Jersey, 07733, USA \\ e-mail: dragan, hchuang, rav, ts2@alcatel-lucent.com
}

\begin{abstract}
In this paper we present a multiuser MIMO experimental system and the corresponding indoor measurement results that demonstrate power of distributed and coherentlycoordinated downlink transmit antennas using zero-forcing (ZF) beamforming. We present experimental performance under different conditions and communication scenarios. We consider different transmit power levels, number of transmit antennas, resolution of channel state information (CSI) quantizer as well as CSI feedback channel data rates. We demonstrate significant gains even in the presence of practical impairments.
\end{abstract}

\section{INTRODUCTION}

In the MIMO broadcast channel (MIMO-BC), a transmitter with multiple antennas sends information over a given bandwidth to multiple receivers. Recent works [1] have shown that the sum capacity of the MIMO-BC can be achieved by means of a nonlinear precoding technique at the transmitter known as dirty paper coding (DPC) [2]. Using channel state information (CSI) and a priori knowledge of the users' data signals, dirty paper coding transmits to multiple users simultaneously so that interference among users' signals is greatly reduced. Unfortunately, this technique has high computational complexity and is difficult to implement in practice.

Beamforming is a simpler, suboptimal technique where signals are simultaneously transmitted to multiple users on beams formed by weighting the phases and amplitudes of the transmitted signals. A particular type of beamforming known as zero-forcing (ZF) uses CSI at the transmitter to form noninterfering beams. It has been shown to achieve the sumcapacity of the MIMO-BC asymptotically as the number of users grows without bound [3] and achieves a significant fraction of the DPC capacity for a finite number of users [4].

ZF beamforming techniques have been considered in wireless cellular communication systems for increasing downlink spectral efficiency. Typically, multiple transmit antennas are co-located at a base station so that beams formed by a given base are non-interfering. However, a user receiving a signal from the beam of one base may experience interference from another base's beam. In channels where the angular spread is sufficiently small, this intercell interference could be reduced by coordinating base stations so that a given area is not simultaneously illuminated by beams from different base stations.
Alternatively, one could perform ZF beamforming over antennas that are spatially distributed throughout the network. If CSI is known throughout the network for each user and the base stations transmit in a coordinated manner, then ideally the users would experience no intercell interference and the spectral efficiency would be greatly improved. A high-speed backhaul network would be required to connect the base stations. Either CSI would need to be transferred among base stations so that the ZF antenna weights are calculated locally at each base, or the CSI would be transferred to a common point where ZF antenna weights are computed and then distributed to each antenna. Realistically, a fully coordinated macro-cellular network would require significant long-term planning. However, near-term applications could include an in-building pico-cell network or a limited outdoor area for hotspot coverage. These applications would be ideally suited for coordinated transmission since the need for spectral efficiency is high and the relatively short distances between base stations makes coordination feasible.

In this paper we describe an experimental prototype that was built for demonstrating ZF beamforming across spatially distributed antennas. In Section II we present the system model. In Section III we describe the experimental system implementation including the transmitter, receiver, and CSI feedback mechanism. In Section IV we present various experimental results. We conclude in Section V.

\section{System MODEL}

We consider a narrowband multiantenna downlink channel modeled as a MIMO-BC with flat fading, where $K$ users, i.e., mobile terminals, each equipped with a single receive antenna, request service from a transmitter with $M$ distributed antennas. The discrete-time complex baseband received signal by the $k$ th user is

$$
y_{k}=\mathbf{h}_{k} \mathbf{x}+n_{k}, \quad k=1, \ldots, K
$$

where $\mathbf{h}_{k}$ is the $k$ th user's $M$-dimensional complex row channel vector, $\mathbf{x}$ is the transmitted signal vector, and $n_{k} \sim \eta(0,1)$ is the complex additive white Gaussian noise with zero mean and unit variance. The noise is uncorrelated among the users but the signal vector is the same for all users. Under zero-forcing beamforming, the transmitted vector $\mathbf{x}=\mathbf{G u}$ where $\mathbf{u}$ is the $K$ - 
dimensional information-bearing signal containing the modulation symbols for each user, and $\mathbf{G}=\mathbf{H}^{H}\left(\mathbf{H H}^{H}\right)^{-1}$ is the $M \times K$ beamforming matrix where $\mathbf{H}$ the $K \times M$ channel matrix. Note that we assumed that the transmitter has perfect knowledge of the CSI. In this case, we can rewrite the received signal by the $k$ th user in (1) as $y_{k}=u_{k}+n_{k}, k=1, \ldots, K$. Hence the rate achieved by this user is simply $\log _{2}\left(1+v_{k}\right)$, where $v_{k}=\mathrm{E}\left[\left|u_{k}\right|^{2}\right]$ is the power allocated to the $k$ th user. The transmit power at the $m$ th antenna is $\left|\mathbf{G}_{m 1}\right|^{2} v_{1}+\ldots+\left|\mathbf{G}_{m K}\right|^{2} v_{K}$. Hence the sum rate optimization subject to a transmit power constraint $P_{m}$ on the $m$ th antenna can be written as:

$$
\begin{aligned}
& \max _{v_{1}, \ldots, v_{K}} \sum_{k=1}^{K} \log \left(1+v_{k}\right), \text { subject to } \\
& \left\{\begin{array}{l}
v_{k} \geq 0, k=1, \ldots, K \\
\sum_{k=1}^{K}\left|\mathbf{G}_{m k}\right|^{2} v_{k} \leq P_{m}, m=1, \ldots, M
\end{array}\right.
\end{aligned}
$$

This optimization has been shown to be a convex optimization [4] which can be solved using conventional interior point techniques.

\section{EXPERIMENTAL SYSTEM}

The experimental system that is used in this study is depicted in Fig. 1. It consists of $M=4$ distributed downlink transmit antennas and $K=2$ mobile terminals, each with a single receive antenna. The transmit antennas are connected to the outputs of the beamformer. The beamformer resides in a base station that determines its coefficients and applies them on the information-bearing signals for each mobile terminal. Using a pilot-assisted channel state estimation, the mobile terminals estimate the downlink channel states between each transmit and receive antenna. The quantized estimates, that correspond to channel state information (CSI), are fed back to the base station, where they are used to determine the beamforming coefficients.

In the following we provide more details on the functional blocks of this experimental system.

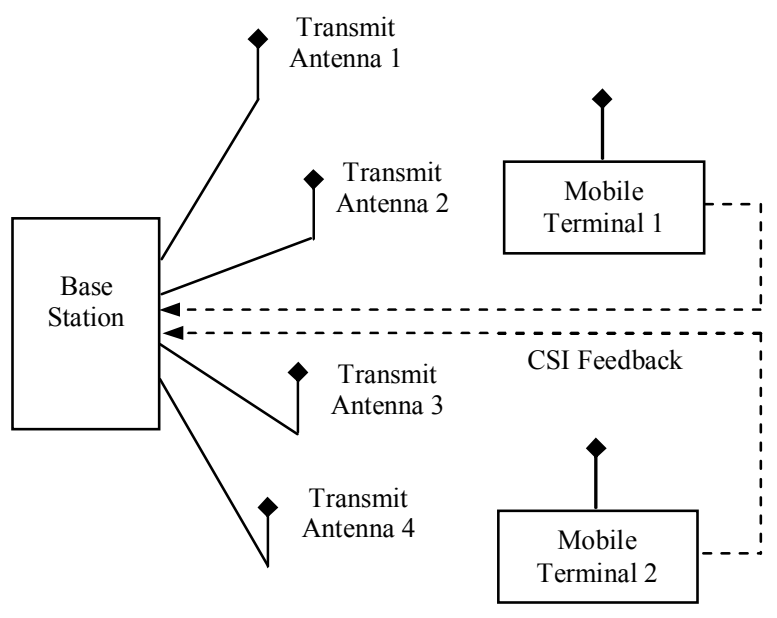

Fig. 1: Experimental system overview.

\section{Base Station}

The functional block diagram of the base station is presented in Fig. 2. The base station receives CSI from the mobile terminals. Specifically, mobile terminal $k$ feeds back estimates of the $k$ th row vector of the MIMO channel matrix $\mathbf{H}$ (for $k=1,2$ ). Having the CSI received, the base station determines the beamforming coefficients.

The output of the generalized beamformer is $\mathbf{x}=\mathbf{G u}$, where $\mathbf{x} \in \mathbb{C}^{4 \times 1}$ and $\mathbf{G} \in \mathbb{C}^{4 \times 2}$ is the beamforming matrix. Furthermore, $\mathbf{u} \in \mathbb{C}^{2 \times 1}$ is the input vector where $u_{k}$ is its $k$ th element and it corresponds to the signal dedicated to mobile terminal $k(k=1$, 2). The signal $u_{k}$ consists of the information-bearing signal as well as dedicated pilot $k$ (i.e., reference signal). Dedicated pilot $k$ allows the mobile terminals to estimate the $k$ th column entries of the composite MIMO channel matrix GH. The particular estimates are used by the mobile terminals to perform coherent detection of the information bearing portion of the signal $u_{k}$ (for $k=1,2$ ) without any explicit knowledge of beamforming that is performed by the base station. To simplify the estimation procedure, the dedicated pilots are orthogonal in signal space to the information-bearing portion of the signals, and to each other.

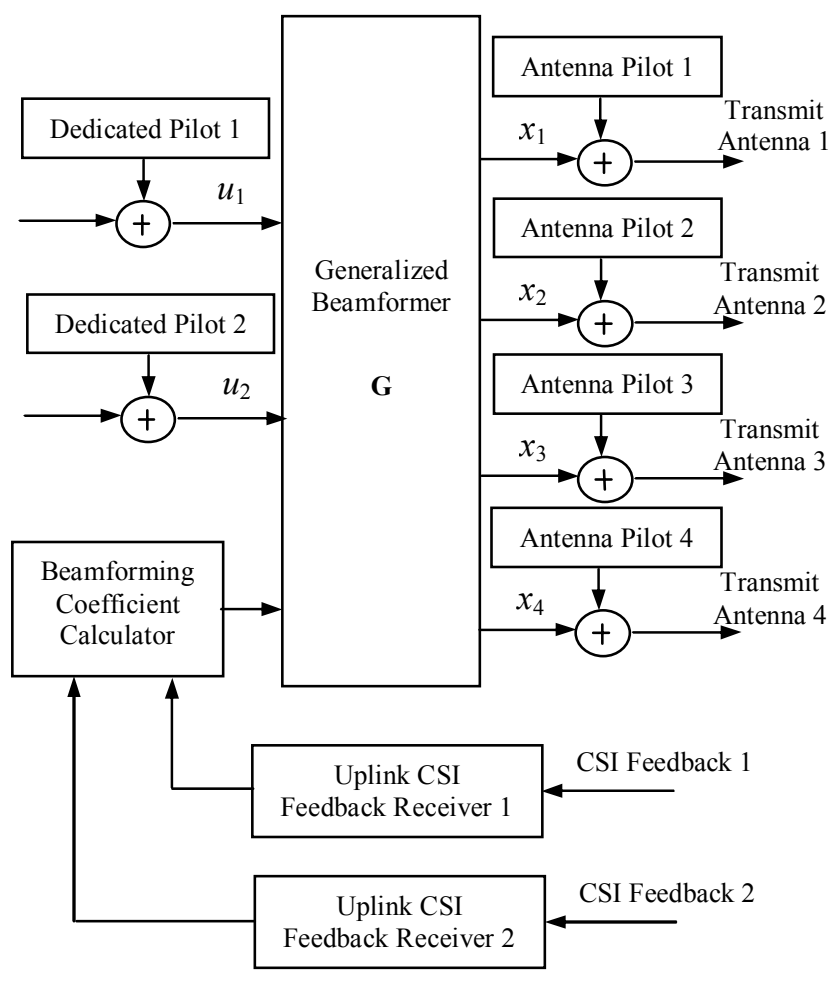

Fig. 2: Base station functional blocks.

Before being transmitted over the corresponding downlink transmit antenna, a unique pilot is added to each beamformer outputs. The antenna-specific pilots allow mobile terminals to perform pilot-assisted estimation of the MIMO channel $\mathbf{H}$. Specifically, mobile terminal $k$ estimates the $k$ th row vector of the matrix $\mathbf{H}$. The quantized estimates correspond to the CSI that is fed back to the base station. To simplify the estimation 
procedure, the antenna-specific pilots are orthogonal in signal space to the beamformer outputs and to each other.

\section{Mobile Terminal}

The functional block diagram of the mobile terminal is presented in Fig. 3. It consists of a number of estimators that implement maximum likelihood estimation [5]. Specifically, in this implementation the mobile terminal functionality is dedicated to assessing the performance of the distributed antenna beamforming.

The estimators that correspond to dedicated pilot 1 and 2 are used to estimate the received power of the signal that is dedicated to mobile terminal 1 and 2 , respectively. $\hat{S}_{k l}$ denotes estimated power of mobile terminal $l$ signal that is received at mobile terminal $k$. For example, in the case when idealized ZF beamforming and estimation are applied, the received power estimates should be $\hat{S}_{12}=\hat{S}_{21}=0$ with $\hat{S}_{11} \geq 0$ and $\hat{S}_{22} \geq 0$, which is a direct consequence of the zero-forcing criterion.

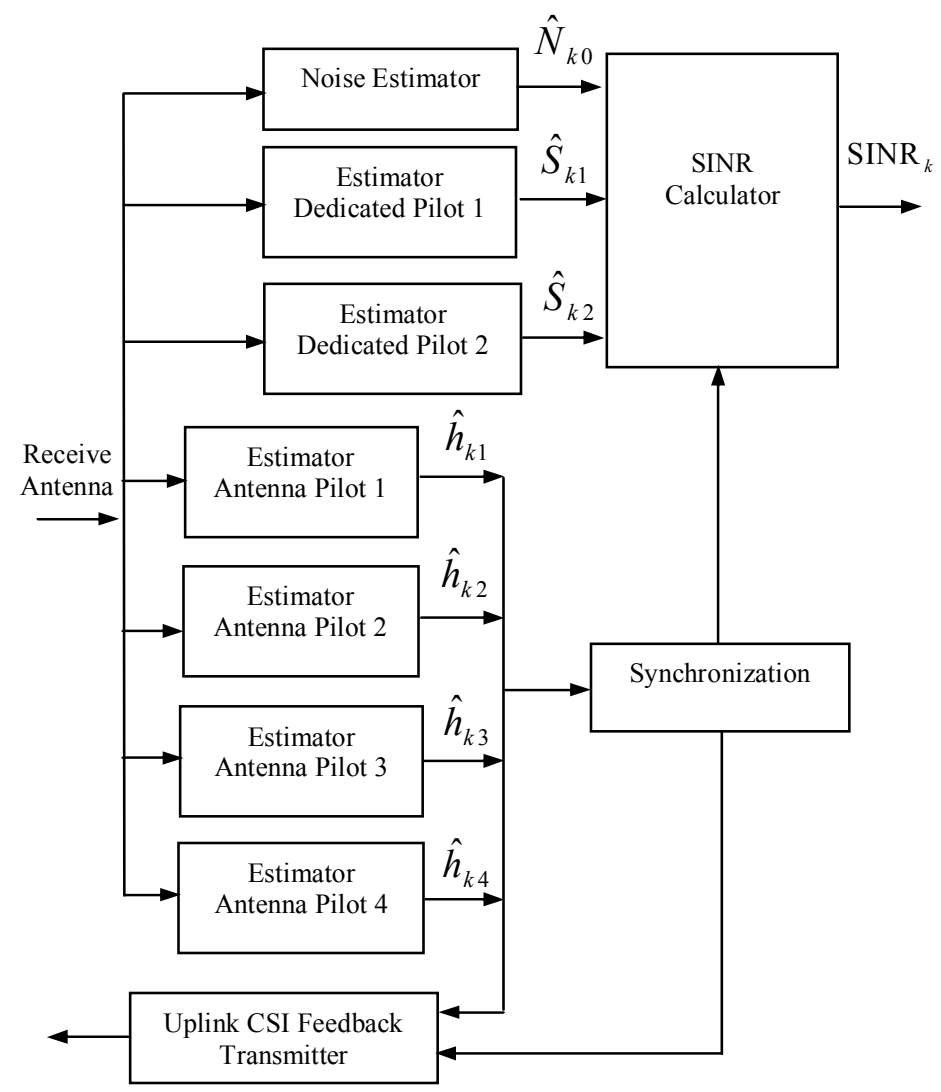

Fig. 3: Mobile terminal functional blocks, $k=1$ or 2 .

As a figure of merit we consider signal-to-noise-plusinterference ratio (SINR)

$$
\operatorname{SINR}_{1}=\frac{\hat{S}_{11}}{\hat{S}_{12}+\hat{N}_{10}} \text { and } \operatorname{SINR}_{2}=\frac{\hat{S}_{22}}{\hat{S}_{21}+\hat{N}_{20}}
$$

where $\hat{N}_{10}$ and $\hat{N}_{20}$ are estimates of the noise power at mobile terminal 1 and 2, respectively.

The antenna-specific pilots are used by the mobile terminals to perform pilot-assisted estimation of the MIMO channel H. As said earlier, mobile terminal $k$ estimates the $k$ th row vector $\hat{\mathbf{h}}_{k}=\left[\hat{h}_{k 1} \ldots \hat{h}_{k 4}\right]$ of the matrix $\mathbf{H}$. The quantized estimates correspond to the CSI that is transmitted back to the base station, where is used to calculate the beamforming coefficients.

\section{Signal Arrangement}

The symbol rate of the experimental system is set to 1.2288 Msym/sec, which is identical to the cdma2000/EV-DO chip rate.

The antenna-specific pilots span 128 symbols, lasting 104.166 usec, and they are code-multiplexed. The dedicated pilots have the identical duration and multiplexing scheme. The pilot codes are mutually orthogonal and quasi-random with a low cross correlation. Immediately after the antenna-specific pilots, the dedicated pilots are transmitted. Furthermore, the information-bearing portion (payload) of the signal follows after the dedicated pilots. The payload duration may vary depending on a particular experimental setup. The temporal relationship between the pilots and the payloads is depicted in Fig. 4.

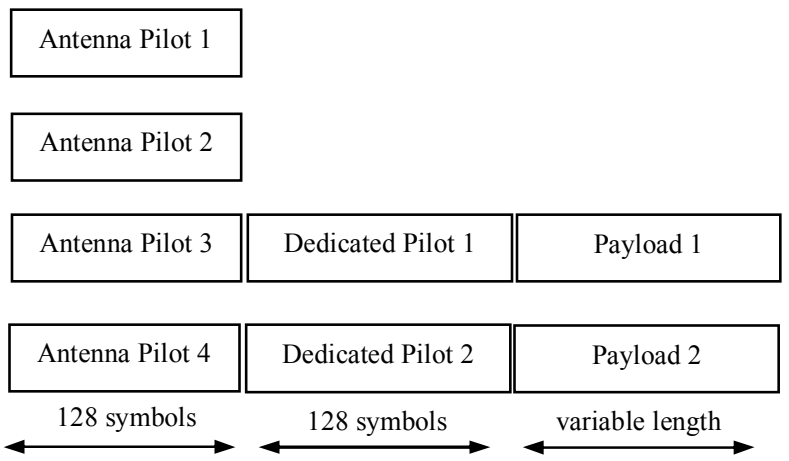

Fig. 4: Temporal relationship between the antenna-specific pilots, dedicated pilots and the payloads.

Note that the beamforming is not performed while the antenna-specific pilots are being transmitted. The beamforming is applied during the transmission of the dedicated pilots and payloads.

\section{Channel State Information Feedback}

Mobile terminal $k$ periodically obtains the channel state estimates $\hat{\mathbf{h}}_{k}=\left[\hat{h}_{k 1} \ldots \hat{h}_{k 4}\right],(k=1,2)$.

The shortest period between new estimates is 256 symbols, i.e., 208.332 usec. The shortest period corresponds the case when no payload is transmitted. 
Before being transmitted on the uplink, each component of the vector $\hat{\mathbf{h}}_{k}$ is quantized with a 15 -bit liner quantizer both for the real $(\mathrm{I})$ and imaginary $(\mathrm{Q})$ component.

The CSI feedback channel is realized over a cable (CAT5), which is a highly controllable medium allowing us to assess the system performance under different CSI feedback channel conditions. In the future we plan to implement a wireless CSI feedback channel.

\section{Implementation Platform and Features}

The most of the functional blocks that are depicted in Fig. 2 and 3 are implemented on a FPGA platform (Xilinx Virtex II 6000 ), using a small fraction of the available resources. Only the beamformer coefficient calculator is implemented on a floating point DSP (TI 6701).

The mobile terminal front-end is based on a heterodyne architecture, followed by a 10-bit analog-to-digital converter. The carrier frequency is set to $2.1 \mathrm{GHz}$.

Furthermore, the downlink transmitters and each mobile terminal have free running oscillators that are manually tuned. The measured frequency offsets and clock drifts are negligible compared to the estimation periods and wireless channel coherence periods.

\section{EXPERIMENTAL RESULTS}

The experimental results that are presented in this section are based on the measurements that are conducted in our laboratory. The laboratory floor plan is given in Fig. 5, where the solid lines correspond to its walls. Typical laboratory furniture (benches and wooden cabinets), computers and instruments occupy the laboratory, thus, creating a rich scattering environment. Each downlink transmit antenna (TX1 to TX4) is placed close to a different corner of the laboratory. The exact positions of the transmit antennas are given in Fig. 5. The height of each transmit antennas is $2 \mathrm{~m}$. During the measurements, the mobile terminals (M1 and M2) are placed randomly within the shaded region in Fig. 5. Per each measurement setup, 100 SINR measurements are recorded each corresponding to different positions of mobile terminal 1 and 2 .

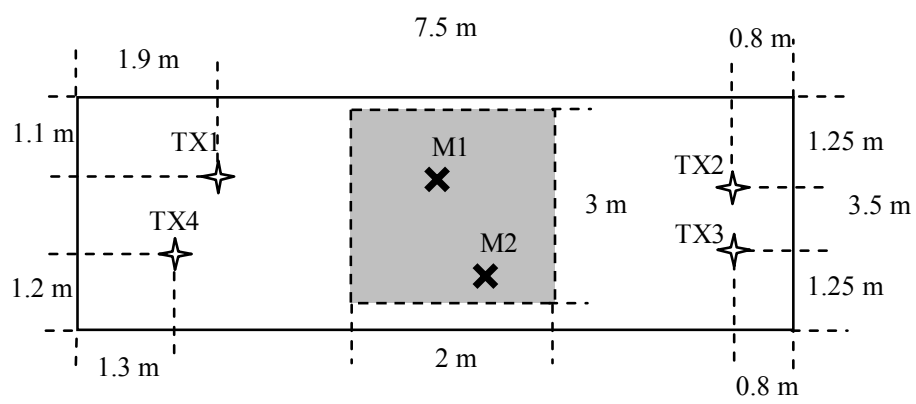

Fig. 5: Laboratory floor plan.

For the results that are presented in this section, the CSI is fed back every $2.08332 \mathrm{msec}$ (480 times per second), corresponding to $5 \%$ overhead for the antenna-specific pilots as well as $5 \%$ overhead for the dedicated pilots. Furthermore, if not stated otherwise, the 15-bit linear quantizer is used for the real (I) and imaginary (Q) component of the channel state estimate.

In all cases the total average transmit power is identical. For example, in a case when the number of transmit antennas is $M$ $=4$ the average transmit power per one antenna is 4 times lower than in a case with the single transmit antenna, $M=1$.

In Fig. 6 we present cumulative distribution function (CDF) of SINR for different transmission schemes. In addition to ZF beamforming that is described in the previous sections, we present a single user case. In the single user case, the information-bearing signal is sent only to one of the users. The signal is sent from transmit antenna 1 (TX1). The single user case would correspond to a time-division multiplexing where transmissions for different users are orthogonalized in time. Furthermore, we considered a case when no beamforming is applied. Specifically, the information-bearing signal for mobile terminal 1 is transmitted only from transmit antenna 1 (TX1). Concurrently, the information-bearing signal for mobile terminal 2 is transmitted only from transmit antenna 2 (TX2). In other words, two independent transmissions are happening at the same time without any interference mitigation scheme being applied.

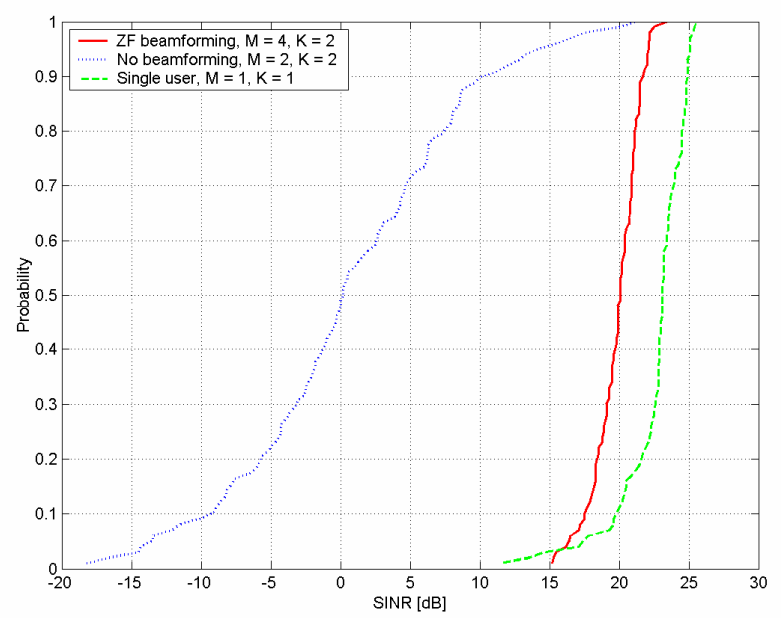

Fig. 6: CDF of SINR for different transmission schemes.

The corresponding CDF of the achievable downlink sum data rates is presented in Fig. 7. The rates account for the pilot overhead. The pilot overhead for ZF beamforming is $10 \%$ while for the other two schemes it is $5 \%$ (because the antennaspecific pilots are not needed). The results demonstrate significant gains for $\mathrm{ZF}$ beamforming.

In Fig. 8 we present CDF of SINR for different levels of the transmit power when ZF beamforming is applied (for $M=4$ and $K=2$ ). Note that even though the transmit power is varied by $10 \mathrm{~dB}$, the change of the measured SINR is smaller. These are the characteristics of an interference-limited system. We believe that this is a consequence of a number of practical impairments. For example, imperfect channel state estimation, CSI quantization and delay in its feedback are some of the impairments leading to the interference-limited behavior [6]. However, even in the presence of the practical impairments, 
based on the results in Fig. 6 and 7, ZF beamforming gains are significant compared to the other solutions that are considered.

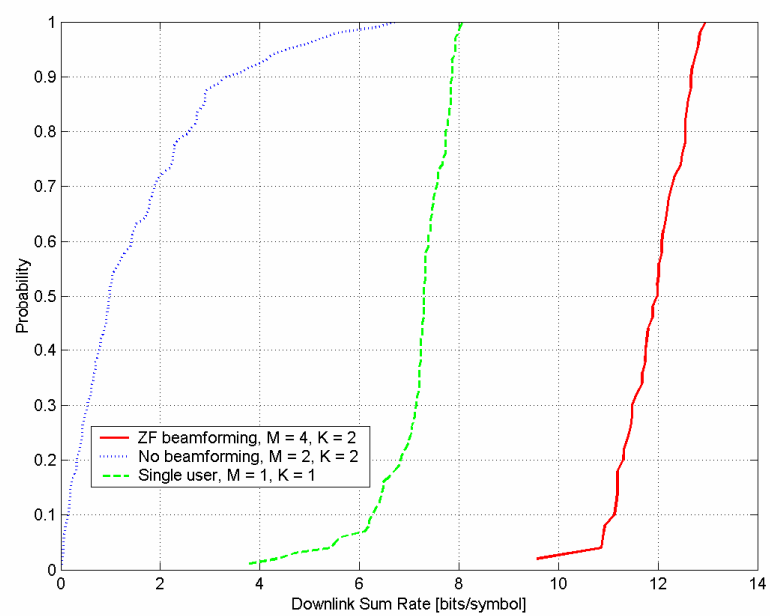

Fig. 7: CDF of downlink sum data rates for different transmission schemes.

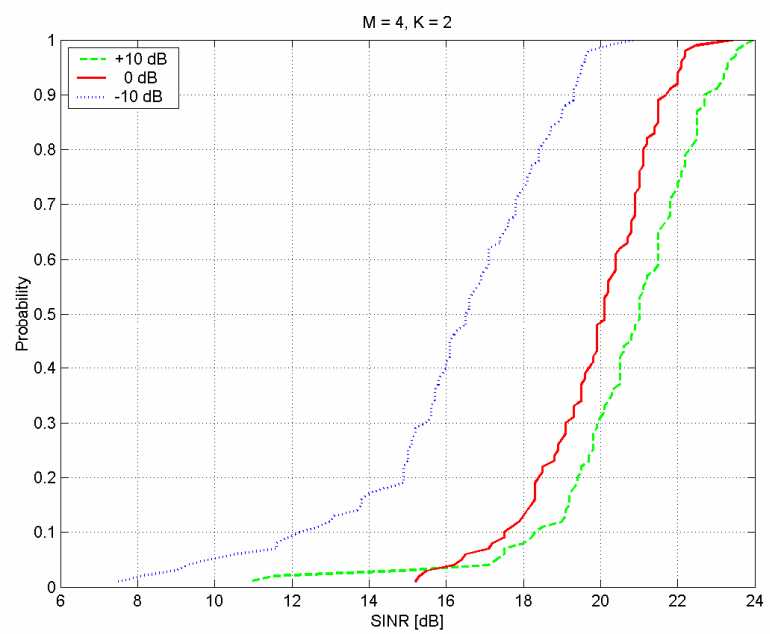

Fig. 8: CDF of SINR for different transmit power levels.

In Fig. $9 \mathrm{CDF}$ of SINR is presented for different number of the downlink transmit antennas and ZF beamforming (for $M=$ 2,3 and 4 and $K=2$ ). As expected, the performance is improved as the number of antennas is increased. As said earlier, in all cases the total average transmit power is identical.

In Fig. $10 \mathrm{CDF}$ of SINR is presented for different resolutions of the CSI quantizer and ZF beamforming (for $M=$ 4 and $K=2$ ). Considering that the CSI is fed back every 2.0833 msec, the presented 15-, 8-, 6- and 4-bit quantizer correspond to the CSI feedback data rates of $57.6 \mathrm{kbps}, 30.72 \mathrm{kbps}, 23.04$ kbps and $15.36 \mathrm{kbps}$, respectively. Note that the applied quantizer is linear and not optimal. Therefore the presented results should be viewed as a lower bound on performance that may be improved if better CSI quantizers are applied.

Let us now consider a tradeoff between the number of transmit antennas and the CSI feedback data rates. For example, based on the results in Fig. 9 and 10 the performance of ZF beamforming with two transmit antennas $M=2$, and the 15-bit quantizer is similar or better than the performance of the same beamforming scheme with four transmit antennas $M=4$, and the 8-bit quantizer. In both cases the required CSI feedback data rate is comparable, i.e., $28.8 \mathrm{kbps}$ versus 30.72 kbps. In other words, we could lower the number of transmit antennas, increase the quantizer resolution, and maintain the performance and the CSI feedback data rates.

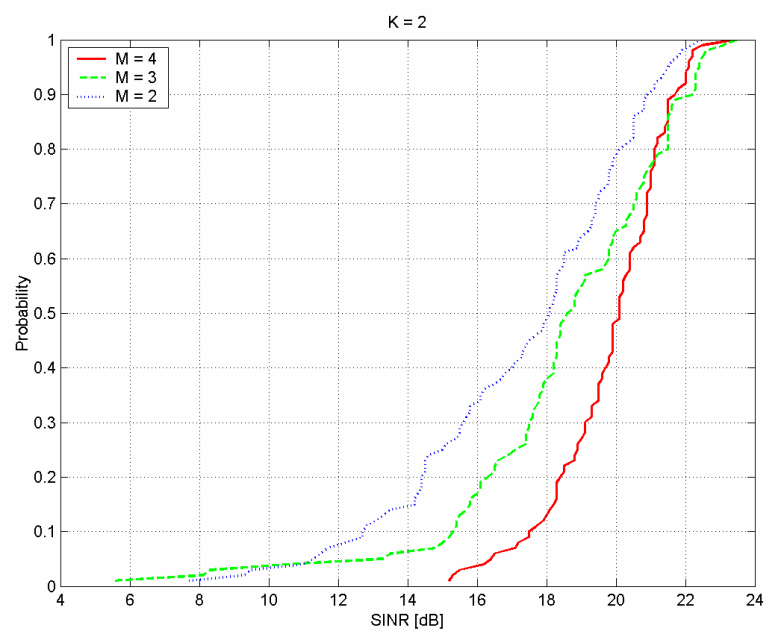

Fig. 9: CDF of SINR for different number of transmit antennas.

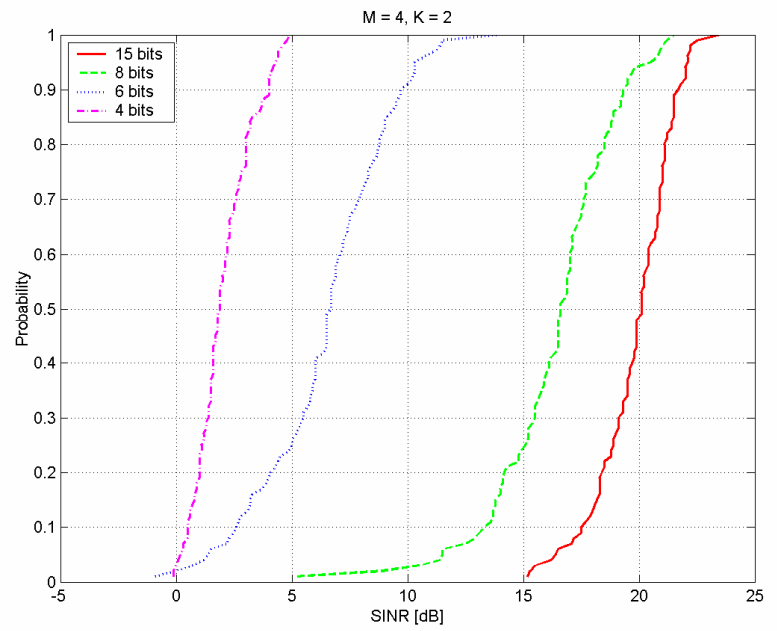

Fig. 10: CDF of SINR for different resolution of the CSI quantizer.

\section{CONCLUSION}

We have presented the multiuser MIMO experimental system and the corresponding indoor measurement results that have demonstrated power of distributed and coherentlycoordinated downlink transmit antennas using ZF beamforming. We have presented experimental performance under different conditions and communication scenarios. We have considered different transmit power levels, number of transmit antennas, resolution of the CSI quantizer as well as the CSI feedback channel data rates. We have demonstrated significant gains even in the presence of practical impairments. 


\section{ACKNOWLEDGEMENTS}

The authors are grateful to Clark Woodworth and Susan Walker for their support during the implementation of the experimental prototype.

\section{References}

[1] H. Weingarten, Y. Steinberg, S. Shamai, "Capacity region of the MIMO broadcast channel," IEEE Trans. Inform. Theory, vol. 52, no. 9, pp. 3936-3964, September 2006.

[2] M. Costa, "Writing on dirty paper," IEEE Trans. Inform Theory, vol. 29, no. 3, pp. 439-441, May 1983.

[3] T. Yoo, A. Goldsmith, "On the optimality of multiantenna broadcast scheduling using zero-forcing beamforming," IEEE J. Select. Areas Commun., vol. 24, no. 3, pp. 528-541, Mar. 2006.

[4] F. Boccardi, H. Huang, "Zero-forcing precoding for the MIMO broadcast channel under per-antenna power constraints," IEEE Workshop on Signal Processing Advances in Wireless Communications, July 2006.

[5] D. Samardzija, N. Mandayam, "Pilot assisted estimation of MIMO fading channel response and achievable data rates," IEEE Trans on Sig. Proc., vol. 51, pp. 2882-2890, Nov. 2003.

[6] D. Samardzija, N. Mandayam, D. Chizhik, "Adaptive transmitter optimization in multiuser multiantenna systems: theoretical limits, effect of delays and performance enhancements," EURASIP Journal on Wireless Communications and Networking, no. 3, pp. 298-307, Aug. 2005 